\title{
On the density of continuous functions in variable exponent Sobolev space
}

Peter A. Hästö

\begin{abstract}
In this article we give new conditions for the density of continuous or smooth functions in variable exponent Sobolev spaces. Our first result combines the previously known sufficient conditions, a monotony condition by Edmunds and Rákosník and a continuity condition independently due to Samko and Diening, into a single weaker condition. The second main result gives a sufficient condition in terms of the regularity of the level-sets of the variable exponent.
\end{abstract}

\section{Introduction}

In this paper we prove the density of smooth or continuous functions in variable exponent Sobolev spaces under new and weaker conditions than were previously known. The first main result is a refinement of previous results; the second is derived using a new method.

Variable exponent Lebesgue and Sobolev spaces have been intensely investigated in the last five year, see $[8,12,20,17,34]$ for overviews. The main motivation for studying these spaces are differential equations with nonstandard growth and coercivity conditions, see e.g. [1, 2, 13, 29], which have been applied in models of electrorheological fluids [32] and image restoration [3]. One major factor in this increase in interest was the discovery of a fairly weak condition on the exponent which guarantees that the variable exponent space has most properties of classical Lebesgue and Sobolev spaces. This is the so-called logarithmic Hölder continuity (a.k.a. weak-Lipschitz or Dini-Lipschitz) condition:

$$
|p(x)-p(y)| \leqslant \frac{C}{-\log |x-y|} \quad \text { for }|x-y| \leq \frac{1}{2} .
$$

2000 Mathematics Subject Classification: Primary: 46E35; Secondary: 26A15.

Keywords: Variable exponent, Sobolev spaces, density of smooth functions, density of continuous functions. 


\section{P. A. НёsтÖ}

One very important consequence of this condition is that the maximal operator is locally bounded in $L^{p(\cdot)}[7]$, see also $[4,30]$. Moreover, the condition is essentially the optimal one for drawing this conclusion [31]. More recently also potential type operators and singular integrals have been studied in variable exponent spaces under the same condition, see e.g., $[6,9,10$, $18,26]$.

In contrast to the situation of the maximal operator, necessary and sufficient conditions for the density of smooth functions in Sobolev space are quite intricate. Samko [33] and Diening [7] have shown, independently, that log-Hölder continuity of the exponent is sufficient for the density of smooth functions. In more general domains, a density result under the log-Hölder assumption was recently obtained by Cruz-Uribe and Fiorenza [5, Theorem 2.6]. Edmunds and Rákosník [11] have given a sufficient condition of a different kind, which requires that the exponent be monotone in a certain sense. Their conditions allows no saddle points, strict minima or maxima, but works even for some discontinuous exponents. ${ }^{1}$

These conditions have some obvious short-comings: the Edmunds-Rákosník monotony condition fails if the exponent has a strict minimum or maximum, no matter how regular it would otherwise be there. The continuity condition, on the other hand, does not work for instance if the exponent is piece-wise constant, a case in which it is often easy to see that we have density. The first result of this paper, Theorem 3.2, combines the sufficient conditions of Samko/Diening and Edmunds-Rákosník to give a single, much weaker condition. Although this new result remedies some of these short-comings, there are still some obvious cases where it does not work. One example is when the exponent has a ridge or trough (see Figure 2 on page 224).

The density results in $[5,7,11,33]$, like Theorem 3.2 , are all based on convolving the Sobolev function with a suitable mollifier. However, this tool does not seem very well suited to variable exponent spaces, since convolution with a smooth compactly supported function is in general not bounded from $L^{p(\cdot)}$ to itself.

In Section 4 we introduce a method which is based on convolution only in the level-sets of the exponent. Once we restrict our attention to the level-set of the exponent, convolution again becomes a very natural operation which does not impose any additional restrictions on the exponent. However, to patch up our approximations on level-sets we have to assume that the levelsets are bilipschitz images of parallel planes or concentric circles.

\footnotetext{
${ }^{1}$ After this article was completed, two more investigations on this issue were completed by Zhikov [37] and Fan, Wang and Zhao [15]. The latter article includes also Theorem 3.2 of this paper.
} 
In contrast to previous results, Theorem 4.10 allows us to conclude only that continuous, but not necessarily smooth, functions are dense.

It is interesting to note that although the level-sets of the exponent seems like a prime candidate for determining whether a variable exponent space is well-behaved or not, there is to-date only one result based on the regularity of level-sets, by Rưzička on the Sobolev embedding theorem [32, Proposition 2.9].

Although it should be clear to anyone studying function spaces that the question of density of continuous or smooth functions is central, it might have some additional ramifications in the variable exponent spaces. Based on earlier work by Zhikov [36], the author presented an example of a variable exponent Sobolev space with uniformly continuous exponent in which continuous functions are not dense and showed that in this space the Dirichlet energy integral minimizer need not be continuous and that not quasievery point need be a Lebesgue point of a Sobolev function [24]. This prompts the question: what happens to the regularity of the variable exponent space if we assume that continuous functions are dense instead of assuming that the exponent is log-Hölder continuous? We return to this and other open questions in Section 5.

\section{Preliminaries}

For $x \in \mathbb{R}^{n}$ and $r>0$ we denote by $B^{n}(x, r)$ the open ball with center $x$ and radius $r$. We abbreviate $B^{n}(r)=B^{n}(0, r), S(r)=\partial B^{n}(r)$ and $B^{n}=B^{n}(1)$. For a ball $B$ and a constant $c$ we denote by $c B$ the ball with the same center as $B$ and $c$ times the radius. By $e_{i}$ we denote the $i^{\text {th }}$ unit vector. By $\Omega$ we always denote a non-empty open subset of $\mathbb{R}^{n}$. The inequality $u \lesssim v$ means that there exists a constant $C$ such that $u \leqslant C v$. For an integrable function defined on a set $A$ of finite non-zero measure we denote

$$
u_{A}=f_{A} u(x) d x=\frac{1}{|A|} \int_{A} u(x) d x .
$$

Let $p: \Omega \rightarrow[1, \infty)$ be a measurable bounded function, called a vari-

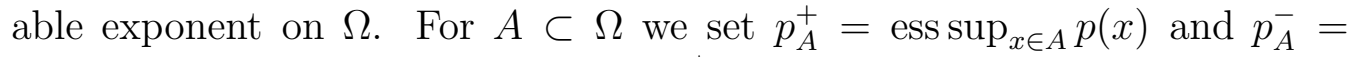

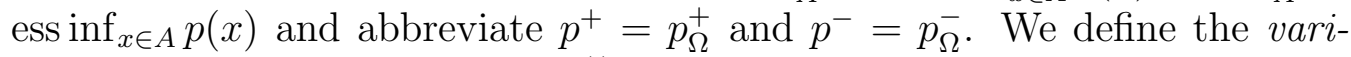
able exponent Lebesgue space $L^{p(\cdot)}(\Omega)$ to consist of all measurable functions $u: \Omega \rightarrow \mathbb{R}$ for which the modular $\varrho_{p(\cdot)}(u)=\int_{\Omega}|u(x)|^{p(x)} d x$ is finite. We define the Luxemburg norm on this space by

$$
\|u\|_{L^{p(\cdot)}(\Omega)}=\|u\|_{p(\cdot)}=\inf \left\{\lambda>0: \varrho_{p(\cdot)}(u / \lambda) \leqslant 1\right\} .
$$


One central property of these spaces (since $p$ is bounded) is that $\varrho_{p(\cdot)}\left(u_{i}\right) \rightarrow 0$ if and only if $\left\|u_{i}\right\|_{p(\cdot)} \rightarrow 0$. This and many other basic results were proven in [28] (similar results were derived earlier in the one-dimensional case by Sharapudinov [35]).

It is also possible to consider variable exponent spaces with unbounded exponents. However, in this case we do not know whether continuous functions are dense in $L^{p(\cdot)}$. Also, functions with compact support need not be dense even in $L^{p(\cdot)}$. Examples of this were given, independently, by CruzUribe \& Fiorenza [5, p. 5] and Harjulehto [19, Example 3.1].

The variable exponent Sobolev space $W^{1, p(\cdot)}(\Omega)$ is the subspace of functions $u \in L^{p(\cdot)}(\Omega)$ whose distributional gradient exists almost everywhere and satisfies $|\nabla u| \in L^{p(\cdot)}(\Omega)$. The norm $\|u\|_{1, p(\cdot)}=\|u\|_{p(\cdot)}+\|\nabla u\|_{p(\cdot)}$ makes $W^{1, p(\cdot)}(\Omega)$ a Banach space. When we want to emphasize in which set the norm is taken we use the notation $\|u\|_{W^{1, p(\cdot)}(\Omega)}$. We also define a modular in the Sobolev space, $\varrho_{1, p(\cdot)}(u)=\varrho_{p(\cdot)}(u)+\varrho_{p(\cdot)}(|\nabla u|)$.

We will derive results for the density of smooth and continuous functions in Sobolev spaces. For an open set $\Omega$ we denote the set of continuous and smooth functions by $C(\Omega)$ and $C^{\infty}(\Omega)$, respectively. By smooth we mean that the classical derivatives of arbitrary order exist at every point of the open set. By $C_{0}(\Omega)$ and $C_{0}^{\infty}(\Omega)$ we denote the corresponding spaces of functions which have compact support in $\Omega$.

\section{A merger of old conditions}

In this section we will show how to combine the continuity condition and the monotony condition to give a single, weaker condition for the density of smooth functions in variable exponent Sobolev space. The new condition says that the exponent need not be monotone, as long as it decreases no more than allowed by log-Hölder continuity.

The idea of the proof of the next lemma is to combine the directed mollifier of Edmunds-Rákosník with an estimate based on the Diening's method from [7].

Lemma 3.1. Suppose that the bounded variable exponent $p$ is defined on $\Omega=B^{n}(7 / 6)$ and let $K \geqslant 0$. Suppose that there exist $r \in(0,1 / 12)$ and $h \in(0,1)$ such that for every $x \in B^{n}$ we have

$$
p(y)-p(x) \geqslant-\frac{K}{\log (1 /|x-y|)}
$$

for every $y$ in the cone

$$
\bigcup_{0<t \leqslant r} B^{n}\left(x+t e_{1}, h t\right) .
$$

Then $C^{\infty}\left(B^{n}\right) \cap W^{1, p(\cdot)}\left(B^{n}\right)$ is dense in $W^{1, p(\cdot)}\left(B^{n}\right)$. 
Proof. Let $\phi \in C_{0}^{\infty}\left(B^{n}\right)$ be a non-negative function of unit integral and fix $\epsilon>0$. For an integrable function $u: B^{n}(7 / 6) \rightarrow \mathbb{R}$ and $\delta \in(0, r)$ we define

$$
R_{\delta} u(x)=\int_{B^{n}} u\left(x+\delta\left(h z+e_{1}\right)\right) \phi(z) d z .
$$

The usual integration-by-parts argument shows that $R_{\delta} u$ is smooth.

Let $u \in L^{p(\cdot)}(\Omega)$. We next show that $\left\|R_{\delta} u-u\right\|_{p(\cdot)} \rightarrow 0$. Recall that to do this it suffices to show that $\varrho_{p(\cdot)}\left(R_{\delta} u-u\right) \rightarrow 0$. Using that $\phi$ is bounded, we estimate

$$
\begin{aligned}
\varrho_{p(\cdot)}\left(R_{\delta} u-u\right) & =\int_{B^{n}}\left|\int_{B^{n}}\left(u\left(x+\delta\left(h z+e_{1}\right)\right)-u(x)\right) \phi(z) d z\right|^{p(x)} d x \\
& \lesssim \int_{B^{n}}\left(\int_{B^{n}}\left|u\left(x+\delta\left(h z+e_{1}\right)\right)-u(x)\right| d z\right)^{p(x)} d x
\end{aligned}
$$

Let us denote $B=B^{n}\left(x+\delta e_{1}, h \delta\right)$. Using Hölder's inequality for the fixed exponent $p_{B}^{-}$and the fact that $\int_{B}|u(z)-u(x)|^{p_{B}^{-}} d z \leqslant 1$ for small enough $\delta$ (uniformly), we find that

$$
\begin{aligned}
\int_{B^{n}}\left(\int_{B^{n}} \mid\right. & \left.u\left(x+\delta\left(h z+e_{1}\right)\right)-u(x) \mid d z\right)^{p(x)} d x \\
& =\int_{B^{n}}\left(\left|B^{n}\right| f_{B}|u(z)-u(x)| d z\right)^{p(x)} d x \\
& \lesssim \int_{B^{n}}\left(f_{B}|u(z)-u(x)|^{p_{B}^{-}} d z\right)^{p(x) / p_{B}^{-}} d x \\
& \lesssim \int_{B^{n}}(h \delta)^{-n p(x) / p_{B}^{-}} \int_{B}|u(z)-u(x)|^{p_{B}^{-}} d z d x
\end{aligned}
$$

We will use the method introduced by Diening to deal with this. By assumption we have

$$
p(y) \geq p(x)-\frac{K}{\log (1 /|x-y|)}
$$

for all $y \in B$. Thus $p$ is "log-Hölder with respect to" $x$, which means (precisely) that $\delta^{\left(p_{B}^{-}-p(x)\right) / p_{B}^{-}}$is bounded by a constant independent of $\delta$ and $x$. (This follows since $p_{B}^{-}-p(x) \geqslant K / \log \delta$.) 
Using this, we continue our previous estimate:

$$
\begin{aligned}
\int_{B^{n}}\left(\int_{B^{n}} \mid\right. & \left.u\left(x+\delta\left(h z+e_{1}\right)\right)-u(x) \mid d z\right)^{p(x)} d x \\
& \lesssim \int_{B^{n}}(h \delta)^{n\left(p_{B}^{-}-p(x)\right) / p_{B}^{-}} f_{B}|u(z)-u(x)|^{p_{B}^{-}} d z d x \\
& \lesssim \int_{B^{n}} \int_{B^{n}}\left|u\left(x+\delta\left(h z+e_{1}\right)\right)-u(x)\right|^{p_{B}^{-}} d z d x \\
& =\int_{B^{n}} \int_{B^{n}}\left|u\left(x+\delta\left(h z+e_{1}\right)\right)-u(x)\right|^{p_{B}^{-}} d x d z .
\end{aligned}
$$

Then we pick up along the track of Edmunds-Rákosník. Since $1+$ $|u(x)|^{p(x)}$ is an integrable function, we can choose $\tau>0$ such that

$$
\int_{V} 1+|u(x)|^{p(x)} d x<\epsilon / 2
$$

for every $V \subset B^{n}$ with $|V|<\tau$. For a fixed $z \in B^{n}$ this implies that

$$
\int_{V} 2+\left|u\left(x+\delta\left(h z+e_{1}\right)\right)\right|^{p\left(x+\delta\left(h z+e_{1}\right)\right)}+|u(x)|^{p(x)} d x<\epsilon,
$$

for $|V|<\tau$, since the translate of $V$ also satisfies $\left|V+\delta\left(h z+e_{1}\right)\right|<\tau$. Since $u$ is measurable there exists, by Luzin's theorem, an open set $U \subset B^{n}(7 / 6)$ such that $u$ is continuous in $B^{n}(7 / 6) \backslash U$ and $|U|<\tau / 2$. By choosing $\delta$ small enough we assume that for all $x, y \in \overline{B^{n}} \backslash U$ with $|x-y|<\delta$ we have $|u(y)-u(x)|<\epsilon$. For $z \in B^{n}$ we denote by $U_{z}$ the set of those points $x \in B^{n}$ for which $x \in U$ or $x+\delta\left(h z+e_{1}\right) \in U$. Note that $\left|U_{z}\right|<\tau$ for every $z$. We find that

$$
\begin{aligned}
& \int_{B^{n}} \int_{B^{n}}\left|u\left(x+\delta\left(h z+e_{1}\right)\right)-u(x)\right|^{p_{B}^{-}} d x d z \\
& \leqslant \int_{B^{n}} \int_{B^{n}} \epsilon^{p_{B}^{-}} d x d z+\int_{B^{n}} \int_{U_{z}}\left|u\left(x+\delta\left(h z+e_{1}\right)\right)-u(x)\right|^{p_{B}^{-}} d x d z \\
& \lesssim \int_{B^{n}} \int_{B^{n}} \max \left\{\epsilon, \epsilon^{p^{+}}\right\} d x d z+\int_{B^{n}} \int_{U_{z}}\left|u\left(x+\delta\left(h z+e_{1}\right)\right)\right|^{p_{B}^{-}}+|u(x)|^{p_{B}^{-}} d x d z \\
& \lesssim \max \left\{\epsilon, \epsilon^{p^{+}}\right\}+\int_{B^{n}} \int_{U_{z}} 2+\left|u\left(x+\delta\left(h z+e_{1}\right)\right)\right|^{p\left(x+\delta\left(h z+e_{1}\right)\right)}+|u(x)|^{p(x)} d x d z \\
& \leqslant \max \left\{\epsilon, \epsilon^{p^{+}}\right\}+\int_{B^{n}} \epsilon d z,
\end{aligned}
$$

where, for the third inequality, we used that

$$
|u(x)|^{p_{B}^{-}} \leqslant 1+|u(x)|^{p(x)}
$$

and similarly for $\left|u\left(x+\delta\left(h z+e_{1}\right)\right)\right|^{p_{B}^{-}}$. Thus we have an upper bound which tends to zero with $\epsilon$. 
To complete the proof of the lemma we still have to show that $\| R_{\delta} u-$ $u \|_{1, p(\cdot)} \rightarrow 0$ for a function $u$ in Sobolev space. This follows easily from the $L^{p(\cdot)}$-result that we just derived, because $\partial_{i}\left[R_{\delta} u\right]=R_{\delta}\left[\partial_{i} u\right]$, where $\partial_{i}$ represents differentiation with respect to the $i^{\text {th }}$ coordinate. Thus the previous argument applies to all the $\partial_{i} u$, which are in $L^{p(\cdot)}(\Omega)$ and therefore $|\nabla u| \in L^{p(\cdot)}(\Omega)$

The idea of the next proof is to patch up the balls from the previous lemma following the proof of [11, Theorem 1].

Theorem 3.2. Let $\Omega \subset \mathbb{R}^{n}$. Suppose that for every point $x \in \Omega$ there are four quantities

$$
r_{x} \in\left(0, \frac{1}{2} \min \{1, d(x, \partial \Omega)\}\right),
$$

$h_{x} \in(0,1), \xi_{x} \in S^{n-1}$ and $K_{x} \in[0, \infty)$ such that for every point $y \in B^{n}\left(x, r_{x}\right)$ we have

$$
p(z)-p(y) \geqslant-\frac{K_{x}}{\log (1 /|y-z|)}
$$

for all points $z$ in the cone

$$
C(y)=\bigcup_{0<t \leqslant r_{x}} B^{n}\left(y+t \xi_{x}, h_{x} t\right) .
$$

Then $C^{\infty}(\Omega) \cap W^{1, p(\cdot)}(\Omega)$ is dense in $W^{1, p(\cdot)}(\Omega)$.

Proof. Let $B_{x}=B\left(x, r_{x} / 10\right)$. By a standard covering theorem we find a countable subfamily consisting of disjoint $B_{i}^{\prime}=B_{x_{i}}$ such that $\bigcup_{i=1}^{\infty} 5 B_{i}^{\prime}=\Omega$. We define $B_{i}=6 B_{i}^{\prime}$ and $B_{i}^{*}=7 B_{i}^{\prime}$. We note that we still have

$$
\bigcup_{i=1}^{\infty} B_{i}=\bigcup_{i=1}^{\infty} \overline{B_{i}^{*}}=\Omega
$$

and we see (by the disjointness of the balls $B_{i}^{\prime}$ ) that any point $x \in \Omega$ is contained in at most $\theta$ of the balls $B_{i}^{*}$. Thus there exists a partition of unity by smooth functions $\phi_{i}$ such that $\phi_{i}$ is supported in $B_{i}$ and $\left|\nabla \phi_{i}\right|$ is bounded by $L_{i} \geqslant 1$.

Fix $u \in W^{1, p(\cdot)}(\Omega)$ and $\epsilon>0$. By Lemma 3.1 we can choose $v_{i} \in$ $W^{1, p(\cdot)}\left(B_{i}\right) \cap C^{\infty}\left(B_{i}\right)$ such that

$$
\left\|u-v_{i}\right\|_{W^{1, p(\cdot)}\left(B_{i}\right)}<2^{-i} \epsilon / L_{i} .
$$

Define $v=\sum \phi_{i} v_{i}$. Since at most finitely many of the $\phi_{i}$ are non-zero in a neighborhood of any point, we see that $v$ is smooth. We easily calculate that $\|u-v\|_{W^{1, p(\cdot)}\left(B_{i}\right)} \leqslant \sum_{i=1}^{\infty}\left\|\phi_{i}\left(u-v_{i}\right)\right\|_{W^{1, p(\cdot)}\left(B_{i}\right)} \leqslant \sum_{i=1}^{\infty}\left(1+L_{i}\right)\left\|u-v_{i}\right\|_{W^{1, p(\cdot)}\left(B_{i}\right)}$ $\leqslant 2 \epsilon$. 
Remark 3.3. Notice that if we set $K_{x} \equiv 0$ in the preceding theorem, then we regain the result of Edmunds and Rákosník. More precisely, that gives a corrected version of the condition in [11]. The version in [11] only assumes $p(x) \leqslant p(z)$ for $z \in C(x)$. To see that the result does not hold in that form we need only consider the example of Zhikov (see [36] or [24]). (The additional assumption is used on line 12 of page 234 of [11]. It is actually clear from the paper that the correct version of the condition was what the authors had in mind. However, since the incorrect version has been quoted in the literature, this remark seems in place.)

A special case of the previous theorem is the following:

Corollary 3.4. Let $r \geq 0$ and monotone in the sense of Edmunds-Rákosnik (i.e. satisfy the condition of the previous theorem with $K_{x} \equiv 0$ ), and let $q$ be a log-Hölder continuous function, both defined on $\Omega$ and such that $p=$ $q+r \geq 1$. Then $C^{\infty}(\Omega) \cap W^{1, p(\cdot)}(Q)$ is dense in $W^{1, p(\cdot)}(\Omega)$.

Example 3.5. Consider the exponent shown in Figure 1: here we have added the log-Hölder continuous function $1+x_{1}+\left|x_{2}\right|\left(x_{i}\right.$ refers to the $i^{\text {th }}$ co-ordinate of $x$ ) to the monotone characteristic function $\chi_{\left\{x \in Q: x_{1}<0\right\}}$ in the unit square $Q$. This exponent satisfies the assumptions of the previous corollary.

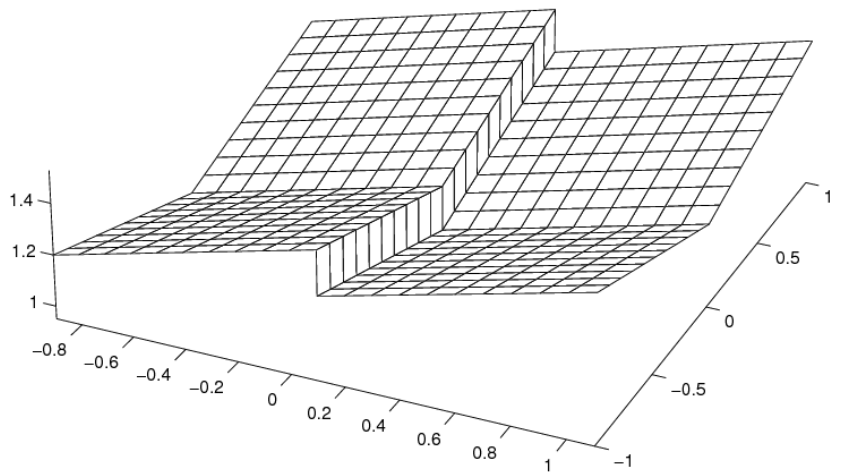

Figure 1: The exponent $p$ which is neither monotone nor continuous at the origin.

As is shown by this corollary and example, the previous theorem is stronger than just saying that the exponent is log-Hölder continuous in one part of the domain and monotone in the rest - the exponent in the example satisfies the assumptions of the theorem only because the exponent is allowed to be either monotone or continuous within a single cone. This flexibility is needed at the origin. 


\section{A new type of condition}

In this section we introduce a new kind of condition for the density of continuous functions in variable exponent Sobolev space. This condition is based on the regularity of the level-sets of the exponent. The proof is composed of two lemmas and corollaries. The idea is to prove the claim in some template cases (a cube and a ball) and then to patch the templates together via bilipschitz mappings.

Lemma 4.1. Let $Q=(-1,1)^{n}$ and $p_{Q}^{+}<\infty$. Suppose that the exponent $p$ depends only on the $n^{\text {th }}$ co-ordinate. Then $C(Q) \cap W^{1, p(\cdot)}(Q)$ is dense in $W^{1, p(\cdot)}(Q)$.

Proof. In this proof $d x, d m_{n-1}(x)$ and $d m_{1}(x)$ stand for integration with respect to the $n-,(n-1)$ - and 1-dimensional Lebesgue measures. We denote the $n^{\text {th }}$ co-ordinate of $x \in \mathbb{R}^{n}$ by $x_{n}$. We use

$$
B=B^{n}(0,1) \cap\left\{x \in \mathbb{R}^{n}: x_{n}=0\right\}
$$

to denote the $(n-1)$-dimensional unit ball which lives in the $x_{n}=0$ plane.

Let $u \in W^{1, p(\cdot)}(Q)$ and assume first that $u$ has compact support in $Q$. We consider only $\epsilon$ smaller than the distance between the support of $u$ and $\partial Q$. Let $\phi: B \rightarrow[0, \infty)$ be smooth and compactly supported with

$$
\int_{B} \phi d m_{n-1}=1
$$

We define an $(n-1)$-dimensional convolution by

$$
u_{\epsilon}(x)=\int_{B} u(x+\epsilon y) \phi(y) d m_{n-1}(y) .
$$

Then clearly $u_{\epsilon}$ is continuous (even smooth) in the plane orthogonal to the $x_{n}$-axis. Consider two points differing in $x_{n}$ co-ordinate. Using that $u$ is absolutely continuous on almost every line parallel to the coordinate axes, we find that

$$
\begin{aligned}
\left|u_{\epsilon}(x)-u_{\epsilon}\left(x+\delta e_{n}\right)\right| & =\left|\int_{B}\left[u(x+\epsilon y)-u\left(x+\delta e_{n}+\epsilon y\right)\right] \phi(y) d m_{n-1}(y)\right| \\
& \lesssim \int_{B}\left|u(x+\epsilon y)-u\left(x+\delta e_{n}+\epsilon y\right)\right| d m_{n-1}(y) \\
& \leqslant \int_{B} \int_{0}^{\delta}\left|\nabla u\left(x+\epsilon y+t e_{n}\right)\right| d m_{1}(t) d m_{n-1}(y) \\
& =\int_{B \times[0, \delta / \epsilon]}|\nabla u(x+\epsilon y)| d y .
\end{aligned}
$$

Since $|\nabla u| \in L^{1}(Q)$, the last integral tends to zero as $\delta \rightarrow 0$. Therefore $u_{\epsilon}$ is uniformly continuous in the $e_{n}$ direction as well, hence in all of $Q$. 
It remains to show that $u_{\epsilon} \rightarrow u$ in Sobolev space. Define $v_{x}(y)=u(x+$ $\epsilon y)-u(x)$ and

$$
M_{\epsilon}(x)=\int_{B}\left|v_{x}(y)\right|^{p(x)} d m_{n-1}(y) .
$$

Using Fubini's theorem for the first equality we find that

$$
\begin{aligned}
\int_{Q} M_{\epsilon}(x) d x & \lesssim \int_{Q} \int_{B}|u(x+\epsilon y)|^{p(x)}+|u(x)|^{p(x)} d m_{n-1}(y) d x \\
& =m_{n-1}(B) \int_{Q}|u(x)|^{p(x)} d x+\int_{B} \int_{Q}|u(x+\epsilon y)|^{p(x)} d x d m_{n-1}(y) \\
& =2 m_{n-1}(B) \varrho_{p(\cdot)}(u) .
\end{aligned}
$$

Thus $M_{\epsilon} \in L^{1}(Q)$. For $M>0$ let $E_{M} \subset Q$ be the set of all $x \in Q$ for which $M_{\epsilon}(x)>M$. The previous inequality also implies that $\left|E_{M}\right| \leqslant C / M$. Choose $M$ so large that $\int_{E_{M}} M_{\epsilon}(x) d m_{n}(x)<\epsilon$.

It also follows that $v_{x} \in L^{p(\cdot)}\left(B, m_{n-1}\right)$ for almost every $x$. Thus we find, using Hölder's inequality for a fixed exponent, that

$$
\begin{aligned}
\int_{Q}\left|u_{\epsilon}(x)-u(x)\right|^{p(x)} d x & =\int_{Q}\left|\int_{B} v_{x}(y) \phi(y) d m_{n-1}(y)\right|^{p(x)} d x \\
& \lesssim \int_{Q} \int_{B}\left|v_{x}(y)\right|^{p(x)} d m_{n-1}(y) d x .
\end{aligned}
$$

We divide this integral into in two parts, and use $\int_{E_{M}} M_{\epsilon}(x) d m_{n}(x)<\epsilon$ :

$$
\int_{Q}\left|u_{\epsilon}(x)-u(x)\right|^{p(x)} d x \lesssim \epsilon+\int_{Q \backslash E_{M}} M_{\epsilon}(x) d x .
$$

For $x \in Q \backslash E_{M}$ we see that $\left\|v_{x}\right\|_{L^{p(\cdot)\left(B, m_{n-1}\right)}}$ is uniformly bounded. Therefore it follows by the continuity of the shift operator (in fixed exponent Lebesgue space) that

$$
\|u(x+\epsilon y)-u(x)\|_{L^{p(y)}\left(B, m_{n-1}\right)} \rightarrow 0
$$

with $\epsilon$ uniformly in $Q \backslash E_{M}$. Thus the second term in (4.2) tends to zero with $\epsilon$, and so $u_{\epsilon} \rightarrow u$ in $L^{p(\cdot)}(\Omega)$.

The approximation result for the gradient is analogous, using the usual identity

$$
\partial_{i} u_{\epsilon}(x)=\int_{B}\left[\partial_{i} u(x+\epsilon y)\right] \phi(y) d m_{n-1}(y) .
$$

Therefore the previous argument applies to all the co-ordinate functions of $\nabla u$, and so $\left\|\nabla\left(u-u_{\epsilon}\right)\right\|_{p(\cdot)} \rightarrow 0$. Thus $u_{\epsilon} \in W^{1, p(\cdot)}(Q) \cap C(Q)$ is the required approximation of the compactly supported function $u$. 
Let then $u \in W^{1, p(\cdot)}(Q)$ be a general, not compactly supported, function. Let $Q_{i}$ be the cube centered at the origin with side-length $2-2^{1-i}$. Define $A_{2}=Q_{2}$ and $A_{i}=Q_{i} \backslash Q_{i-2}$ for larger $i$. Then we can find a partition of unity by Lipschitz functions $\phi_{i}$ such that $\phi_{i}$ is compactly supported in $A_{i}$. Let $L_{i} \geqslant 1$ be the Lipschitz constant of $\phi_{i}$. The function $\phi_{i} u$ has compact support in $Q$, so the previous argument implies that there exists $v_{i} \in Q$ supported in $A_{i}$ such that $\left\|\phi_{i} u-v_{i}\right\|_{1, p(\cdot)} \leqslant 2^{-i} \epsilon / L_{i}$. Then $v=\sum_{i=2}^{\infty} v_{i}$ is continuous and

$$
\|u-v\|_{1, p(\cdot)} \leqslant \sum_{i=2}^{\infty}\left\|\phi_{i} u-v_{i}\right\|_{1, p(\cdot)} \leqslant \sum_{i=2}^{\infty}\left(1+L_{i}\right) 2^{-i} \epsilon / L_{i} \leqslant 2 \epsilon .
$$

Remark 4.3. If $p^{-}>1$, then we can say a bit more about the continuity of $u_{\epsilon}$ : in the previous proof we derived the estimate

$$
\left|u_{\epsilon}(x)-u_{\epsilon}\left(x+\delta e_{n}\right)\right| \lesssim \int_{B \times[0, \delta / \epsilon]}|\nabla u(x+\epsilon y)| d y .
$$

Since $|\nabla u| \in L^{p^{-}}(Q)$, this implies, by Hölder's inequality, that

$$
\begin{aligned}
\mid u_{\epsilon}(x) & -u_{\epsilon}\left(x+\delta e_{n}\right) \mid \\
& \lesssim|B \times[0, \delta / \epsilon]|^{1-1 / p^{-}}\left(\int_{B \times[0, \delta / \epsilon]}|\nabla u(x+\epsilon y)|^{p^{-}} d y\right)^{1 / p^{-}} \lesssim\left(\frac{\delta}{\epsilon}\right)^{1-1 / p^{-}},
\end{aligned}
$$

so that $u_{\epsilon}$ is $\left(1-1 / p^{-}\right)$-Hölder continuous in compact subsets of $Q$.

By a simple trick we can apply the previous lemma to a much wider range of situations. Recall that the mapping $f: Q \rightarrow \mathbb{R}^{n}$ is said to be L-bilipschitz if

$$
\frac{1}{L}|x-y| \leqslant|f(x)-f(y)| \leqslant L|x-y|
$$

for all $x, y \in Q$.

Corollary 4.4. Let $Q=(0,1)^{n}$ and let $f: Q \rightarrow \mathbb{R}^{n}$ be L-bilipschitz. Let $p^{\prime}: Q \rightarrow[1, \infty)$ be a bounded variable exponent which depends only on the $n^{\text {th }}$ coordinate. Define $\Omega=f(Q)$ and $p=p^{\prime} \circ f^{-1}$. Then $C(\Omega) \cap W^{1, p(\cdot)}(\Omega)$ is dense in $W^{1, p(\cdot)}(\Omega)$.

Proof. This is just a change of variables. Let $\epsilon>0, u \in W^{1, p(\cdot)}(\Omega)$ and define $u^{\prime}=u \circ f$. We have

$$
\begin{aligned}
\varrho_{1, p^{\prime}(\cdot)}\left(u^{\prime}\right) & =\int_{Q}\left|u^{\prime}(y)\right|^{p^{\prime}(y)}+\left|\nabla u^{\prime}(y)\right|^{p^{\prime}(y)} d y \\
& \leqslant \int_{\Omega}\left(|u(x)|^{p(x)}+|L \nabla u(x)|^{p(x)}\right) J_{f^{-1}}(x) d x \leqslant L^{n+p^{+}} \varrho_{1, p(\cdot)}(u),
\end{aligned}
$$

so that $u^{\prime} \in W^{1, p^{\prime} \cdot(\cdot)}(Q)$. By the previous lemma we find a function $v^{\prime} \in$ $C(Q) \cap W^{1, p^{\prime}(\cdot)}(Q)$ such that $\varrho_{1, p^{\prime}(\cdot)}\left(u^{\prime}-v^{\prime}\right)<\epsilon$. Define $v=v^{\prime} \circ f^{-1}$. Then by a similar calculation $\varrho_{1, p(\cdot)}(u-v)<L^{n+p^{+}} \epsilon$. 
The previous corollary nicely takes care of the exponents with ridges or troughs:

Example 4.5. Let $Q=(-1,1)^{2}$ and define

$$
p(x)=2-\left(\log \left(100 /\left|x_{2}\right|\right)\right)^{-a}
$$

for some $a>1$. This exponent is shown in Figure 2, to the left. The previous lemma allows us to conclude that continuous functions are dense in $W^{1, p(\cdot)}(Q)$, whereas Theorem 3.2 is not applicable. The graph on right in Figure 2 shows an exponent for which Corollary 4.4 is applicable.
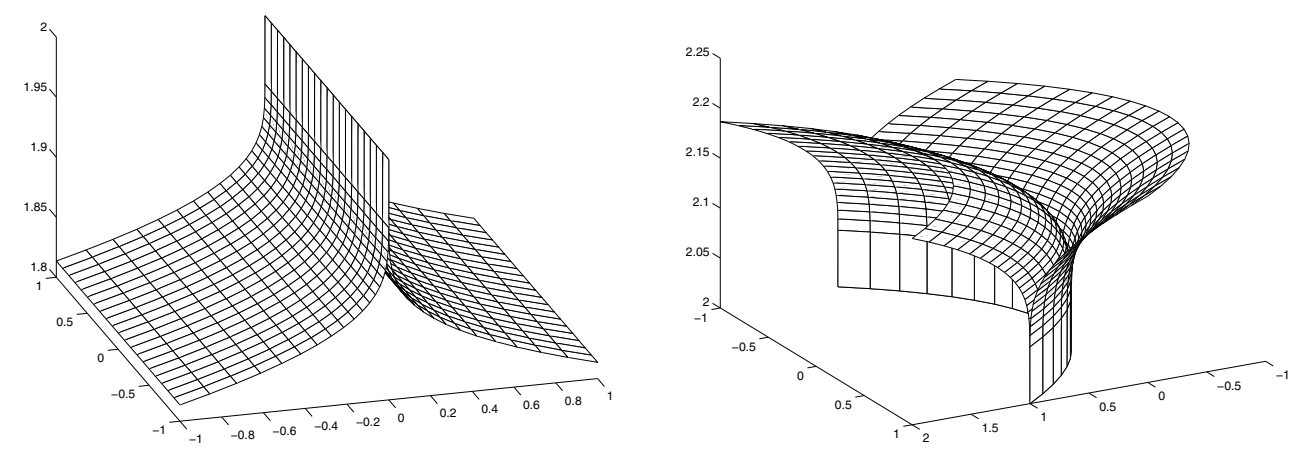

Figure 2: The exponent $p$ with a ridge or trough.

The problem with Corollary 4.4 is that it again does not allow us to to say anything in the case of exponents with strict local minima or maxima. For that we need a different model.

Lemma 4.6. Suppose that the bounded exponent $p$ depends only on $|x|$ in $B^{n}$. Then $C\left(B^{n}\right) \cap W^{1, p(\cdot)}\left(B^{n}\right)$ is dense in $W^{1, p(\cdot)}\left(B^{n}\right)$.

Proof. Let $u \in W^{1, p(\cdot)}(Q)$ and define

$$
v(x)=f_{S(|x|)} u(y) d m_{n-1}(y) .
$$

Using the fixed-exponent Hölder inequality we find that

$$
\int_{S(r)}|v(y)|^{p(y)} d m_{n-1}(y) \lesssim \int_{S(r)}|u(y)|^{p(y)} d m_{n-1}(y)
$$

for $r \in(0,1)$. Integrating this over $r$ implies that $v \in L^{p(\cdot)}\left(B^{n}\right)$. It is easy to see that

$$
\nabla v(x)=f_{S(|x|)} \nabla u(y) \cdot \frac{y}{|y|} d m_{n-1}(y)
$$


so that

$$
|\nabla v(x)| \leq f_{S(|x|)}|\nabla u(y)| d m_{n-1}(y)
$$

Therefore a similar argument shows that $|\nabla v| \in L^{p(\cdot)}\left(B^{n}\right)$.

Fix $\epsilon \in(0,1)$ and choose $r<1$ so small that

$$
\varrho_{W^{1, p(\cdot)}\left(B^{n}(r)\right)}(u)<\epsilon \quad \text { and } \quad \varrho_{W^{1, p(\cdot)}\left(B^{n}(r)\right)}(v)<\epsilon
$$

Consider the domain $D=B^{n} \backslash \overline{B^{n}}(r / 2)$. Denote by $H_{i}^{+}$and $H_{i}^{-}$the sets $\left\{x \in D: x_{i}>0\right\}$ and $\left\{x \in D: x_{i}<0\right\}$, respectively, where $x_{i}$ is the $i^{\text {th }}$ coordinate of $x$. Then each $H_{i}^{ \pm}$satisfies the assumptions of Corollary 4.4 and so we can find continuous functions $w_{i}^{ \pm}$with

$$
\left\|u-w_{i}^{+}\right\|_{W^{1, p(\cdot)}\left(H_{i}^{+}\right)}<\epsilon r \quad \text { and } \quad\left\|u-w_{i}^{-}\right\|_{W^{1, p(\cdot)}\left(H_{i}^{-}\right)}<\epsilon r .
$$

Let $\phi$ be a $(C / r)$-Lipschitz function such that $0 \leqslant \phi \leqslant 1, \phi=1$ on $B^{n}(r / 2)$ and $\phi=0$ on $B^{n} \backslash B^{n}(r)$. Let $\phi_{i}^{ \pm}$be $(C / r)$-Lipschitz functions with support in $H_{i}^{ \pm}$, such that $\left\{\phi, \phi_{1}^{+}, \ldots, \phi_{n}^{-}\right\}$is a partition of unity on $B^{n}$. Define

$$
f=\phi v+\sum_{i=1}^{n} \phi_{i}^{+} w_{i}^{+}+\phi_{i}^{-} w_{i}^{-}
$$

Then we have

$$
\|f-u\|_{1, p(\cdot)} \leqslant\|\phi(v-u)\|_{1, p(\cdot)}+\sum_{i=1}^{n}\left(\left\|\phi_{i}^{+}\left(w_{i}^{+}-u\right)\right\|_{1, p(\cdot)}+\left\|\phi_{i}^{-}\left(w_{i}^{-}-u\right)\right\|_{1, p(\cdot)}\right) .
$$

For the terms in the sum we get

$$
\begin{aligned}
& \left\|\phi_{i}^{ \pm}\left(w_{i}^{ \pm}-u\right)\right\|_{1, p(\cdot)} \\
& \leqslant\left\|w_{i}^{ \pm}-u\right\|_{L^{p(\cdot)}\left(H_{i}^{ \pm}\right)}+\left\|\left(w_{i}^{ \pm}-u\right) \nabla \phi_{i}^{ \pm}\right\|_{L^{p(\cdot)}\left(H_{i}^{ \pm}\right)}+\left\|\nabla\left(w_{i}^{ \pm}-u\right)\right\|_{L^{p(\cdot)}\left(H_{i}^{ \pm}\right)} \\
& \leqslant\left\|w_{i}^{ \pm}-u\right\|_{W^{1, p(\cdot)}\left(H_{i}^{ \pm}\right)}+\frac{C}{r}\left\|w_{i}^{ \pm}-u\right\|_{L^{p(\cdot)}\left(H_{i}^{ \pm}\right)} \leqslant \epsilon r(1+C / r) \lesssim \epsilon .
\end{aligned}
$$

So it remains to estimate $\|\phi(v-u)\|_{1, p(\cdot)}$. To estimate the $L^{p}(\cdot)$-modular of the function is easy:

$$
\begin{aligned}
\int_{B^{n}(r)}|\phi(x)(v(x)-u(x))|^{p(x)} d x & \leqslant 2^{p^{+}-1}\left(\varrho_{L^{p(\cdot)}\left(B^{n}(r)\right)}(u)+\varrho_{L^{p(\cdot)}\left(B^{n}(r)\right)}(v)\right) \\
& <2^{p^{+}} \epsilon .
\end{aligned}
$$


For the gradient we calculate

$$
\begin{aligned}
& \int_{B^{n}(r)} \mid \nabla\left(\left.\phi(x)(v(x)-u(x))\right|^{p(x)} d x\right. \\
& \lesssim \int_{B^{n}(r)}(|\nabla \phi(x)||v(x)-u(x)|)^{p(x)}+|\nabla(v(x)-u(x))|^{p(x)} d x \\
& \lesssim \int_{B^{n}(r)}\left(\frac{2}{r}|v(x)-u(x)|\right)^{p(x)} d x+\varrho_{L^{p(\cdot)}(B(r))}(|\nabla v|)+\varrho_{L^{p(\cdot)}(B(r))}(|\nabla u|) \\
& \leqslant \int_{B^{n}(r)}\left(\frac{2}{r}|v(x)-u(x)|\right)^{p(x)} d x+2 \epsilon .
\end{aligned}
$$

By the $(n-1)$-dimensional, fixed-exponent Poincaré inequality on the sphere $S$ we conclude that

$$
\left(\int_{S}\left(\frac{1}{\operatorname{diam} S}\left|u-u_{S}\right|\right)^{q} d m_{n-1}\right)^{1 / q}=\frac{1}{\operatorname{diam} S}\left\|u-u_{S}\right\|_{L^{q}\left(S, m_{n-1}\right)} \lesssim\|\nabla u\|_{L^{q}\left(S, m_{n-1}\right)},
$$

where $u_{S}$ denotes the $(n-1)$-dimensional average of $u$ over $S$. Let us denote by $\nabla_{S}$ the non-radial gradient, and note that $\nabla_{S} v=0$, since $v$ is radial. Using the Poincaré inequality for the second inequality we find that

$$
\begin{aligned}
\int_{B^{n}(r)}\left(\frac{2}{r}|v(x)-u(x)|\right)^{p(x)} & d x \\
& \lesssim \int_{0}^{r} \int_{S(t)}\left(\frac{1}{t}|v(y)-u(y)|\right)^{p(t)} d m_{n-1}(y) d m_{1}(t) \\
& \lesssim \int_{0}^{r} \int_{S(t)}\left|\nabla_{S}(v(y)-u(y))\right|^{p(t)} d m_{n-1}(y) d m_{1}(t) \\
& \leqslant \int_{0}^{r} \int_{S(t)}|\nabla(v(y)-u(y))|^{p(t)} d m_{n-1}(y) d m_{1}(t) \\
& =\int_{B^{n}(r)}|\nabla(v(x)-u(x))|^{p(x)} d x \lesssim \epsilon .
\end{aligned}
$$

Using this in the previous estimate we conclude that

$$
\varrho_{p(\cdot)}(\nabla(\phi(v-u))) \lesssim \epsilon .
$$

Therefore we have shown that $f \rightarrow u$ in $W^{1, p(\cdot)}\left(B^{n}\right)$.

It remains to show that $f$ is continuous. Now the function $w_{i}^{ \pm}$and $\phi$ are clearly continuous, so we need only worry about $v$. Since $v$ is radial and $v \in W^{1, p(\cdot)}\left(B^{n}\right)$, it behaves much like a one-dimensional Sobolev function away from the origin. Thus we find that

$$
\begin{aligned}
\left|v\left(s e_{1}\right)-v\left(t e_{1}\right)\right| \leqslant \int_{s}^{t}\left|\nabla v\left(r e_{1}\right)\right| d r & \leqslant \frac{1}{s^{n-1}} \int_{s}^{t}\left|\nabla v\left(r e_{1}\right)\right| r^{n-1} d r \\
& =\frac{C}{s^{n-1}} \int_{B^{n}(t) \backslash B^{n}(s)}|\nabla v(x)| d x,
\end{aligned}
$$


for every $0<s<t<1$. We conclude that $v$ is continuous except possibly at the origin. Let $\delta>0$. Then the function

$$
v_{\delta}(x)= \begin{cases}v(x), & \text { for } x \in B^{n} \backslash \overline{B^{n}}(\delta) \\ v\left(\delta e_{1}\right), & \text { for } x \in B^{n}(\delta)\end{cases}
$$

is continuous in $B^{n}$ and approximates $v$ in $W^{1, p(\cdot)}\left(B^{n}\right)$. Using this function in place of $v$, we get a sequence of continuous functions $f_{\delta} \rightarrow f$, so we are done.

Remark 4.7. The previous proof illustrates the use of Corollary 4.4. We can get arbitrarily close to the center-point, but we cannot quite reach it. We return to this question in the next section, see Example 5.3. Note that the function $v$ was necessary, since we do not have a Poincaré inequality at our disposal, unless $p$ is log-Hölder continuous, see [21].

Example 4.8. Define

$$
p(x)=2-(\log (1 /|x|))^{-a}
$$

for some $a>1$. Then the previous lemma allows us to conclude that continuous functions are dense in $W^{1, p(\cdot)}\left(B^{n}\right)$, whereas Theorem 3.2 is not applicable. Another example is given by

$$
p(x)=2+\sin (1 /|x|) .
$$

Variable exponent spaces with exponent depending only on the distance to the origin have been studied previously by Fan, Zhao and Zhao [14] in connection with Strauss-Lion type imbeddings. However, for us the main reason for looking at such spaces is the following corollary and its implications.

Corollary 4.9. Let $f: B^{n} \rightarrow \mathbb{R}^{n}$ be L-bilipschitz. Let $p^{\prime}: B^{n} \rightarrow[1, \infty)$ be a bounded exponent which depends only on $|x|$. Define $\Omega=f\left(B^{n}\right)$ and $p=p^{\prime} \circ f^{-1}$. Then $C(\Omega) \cap W^{1, p(\cdot)}(\Omega)$ is dense in $W^{1, p(\cdot)}(\Omega)$.

The proof is similar to the proof of Corollary 4.4, and is hence omitted.

We can combine the results from the corollaries in this section into the following theorem. Notice that the partition of unity need not be uniformly bilipschitz. This means that we can also handle cases where the regularity of the exponent decreases towards the boundary of the domain.

The statement of the following theorem is quite complicated, but the intuition behind it is simple. We must be able to split the domain into regular pieces with sufficient overlap, such that every piece comes from one of the previous corollaries. 
Theorem 4.10. Let $\Omega \subset \mathbb{R}^{n}$ be open and let $\left\{\Omega_{i}\right\}$ be an open covering of $\Omega$ with a subordinate partition of unity by bilipschitz functions $\phi_{i}$ such that the number of indices $i$ for which $\phi_{i}(x)>0$ is locally bounded. Suppose further that for every $i$ the set $\Omega_{i}$ satisfies the conditions of Corollary 4.4 or 4.9. Then $C(\Omega) \cap W^{1, p(\cdot)}(\Omega)$ is dense in $W^{1, p(\cdot)}(\Omega)$.

Proof. Denote the bilipschitz constant of $\phi_{i}$ by $L_{i}$. Fix $u \in W^{1, p(\cdot)}(\Omega)$ and $\epsilon>0$. By Corollary 4.4 or 4.9 we conclude that $C\left(\Omega_{i}\right)$ is dense in $W^{1, p(\cdot)}\left(\Omega_{i}\right)$. Therefore we can choose $v_{i} \in C\left(\Omega_{i}\right) \cap W^{1, p(\cdot)}\left(\Omega_{i}\right)$ so that

$$
\left\|u-v_{i}\right\|_{W^{1, p(\cdot)}\left(\Omega_{i}\right)} \leqslant \epsilon 2^{-i} / L_{i} .
$$

Then $v=\sum_{i=1}^{\infty} \phi_{i} v_{i}$ is continuous and satisfies

$$
\|u-v\|_{p(\cdot)} \leqslant \sum_{i=1}^{\infty}\left\|\phi_{i}\left(u-v_{i}\right)\right\|_{W^{1, p(\cdot)}\left(\Omega_{i}\right)} \leqslant \sum_{i=1}^{\infty}\left(1+L_{i}\right)\left\|u-v_{i}\right\|_{W^{1, p(\cdot)}\left(\Omega_{i}\right)} \leqslant \epsilon .
$$

It is not quite clear how the conditions of this theorem and those of Theorem 3.2 are related. Theorem 3.2 can handle some cases where the level-sets have cusps, as long as the cusp points in the right direction, see Figure 3. Theorem 4.10, on the other hand, works in cases where there is a cusp or ridge in the $p$-direction, like in Figure 2. (Obviously we can trivially combine the theorems by allowing some of the $\Omega_{i}$ 's in Theorem 4.10 to satisfy Theorem 3.2.)
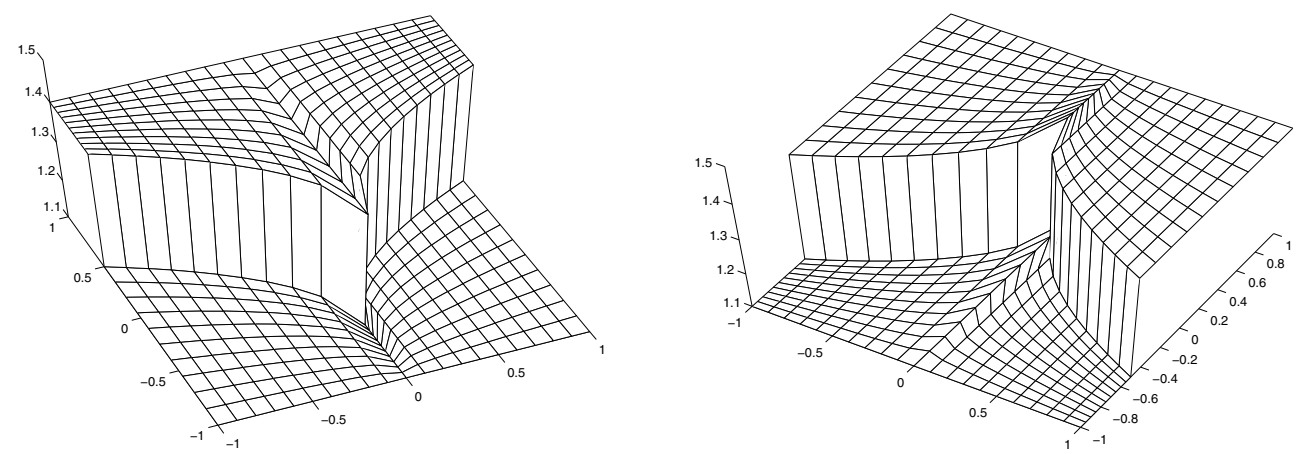

Figure 3: Theorem 3.2 works for the exponent on the left, but not for the exponent on the right.

Remark 4.11. If the bilipschitz mappings in Theorem 4.10 could be replaced by homeomorphisms, then the result would be essentially sharp, since it would cover all cases except when the exponent has a saddle-point. 


\section{Conclusions and questions}

In this article we have given several conditions for the density of continuous or smooth functions in variable exponent Sobolev spaces. Although these conditions are not that simple, they demonstrate that the density of continuous functions is a lot more common occurrence in Sobolev space than the boundedness of the maximal function.

The results in this paper allow us to take care of a variety of exponents. However, if the exponent has a saddle-point, then only the most restrictive condition, log-Hölder continuity, works. On the other hand the example in [24] showed that the growth rate at a saddle-point is critical to whether continuous functions are dense. This raises the following question:

Question 5.1. Suppose that $p: \Omega \rightarrow[1, \infty)$ is a variable exponent without saddle-points. Is $C(\Omega) \cap W^{1, p(\cdot)}(\Omega)$ dense in $W^{1, p(\cdot)}(\Omega)$ ?

Another new aspect that has arisen in this article is that under some conditions we can conclude that continuous (or Hölder continuous) functions are dense in Sobolev space. It is natural to ask whether there in fact exists an exponent such that continuous functions are dense, but smooth functions are not? Intuitively, the converse seems more probable:

Question 5.2. Suppose that $C(\Omega) \cap W^{1, p(\cdot)}(\Omega)$, or $C^{\alpha}(\Omega) \cap W^{1, p(\cdot)}(\Omega)$, is dense in $W^{1, p(\cdot)}(\Omega)$. Is it then true that smooth functions are dense in $W^{1, p(\cdot)}(\Omega)$ ?

If $p^{-}>n$, then every $u \in W^{1, p(\cdot)}(\Omega)$ is continuous (when redefined on a set of zero measure). Therefore a particular case of the previous question is:

Question 5.2'. If $p^{-}>n$, are smooth functions dense in $W^{1, p(\cdot)}(\Omega)$ ?

Harjulehto and Hästö [20] have shown that smooth functions are dense on open intervals of the real line irrespective of the variation of the bounded exponent, so the answer to this question is yes in the case $n=1$.

Arguing as in the proof of Lemma 4.6 it is sometimes possible to exclude a point of irregularity in the domain, if we have a Poincaré inequality at our disposal:

Example 5.3. Consider the square $Q=(-1,1)^{2}$ and define $p$ to equal $p_{1} \in(1,3 / 2)$ below the curve $y=\sqrt{x}$ and $p_{2} \in\left(p_{1}, 3 / 2\right)$ above it, see Figure 3, right. Then

$$
C^{\infty}(Q) \cap W^{1, p(\cdot)}(Q) \quad \text { is dense in } \quad W^{1, p(\cdot)}(Q) .
$$


Proof. It suffices to prove the claim for a bounded function $u \in W^{1, p(\cdot)}(Q)$. For every $r \in(0,1)$ we conclude by Theorem 3.2 that $C^{\infty}\left(Q \backslash B^{n}(r)\right) \cap$ $W^{1, p(\cdot)}\left(Q \backslash B^{n}(r)\right)$ is dense in $W^{1, p(\cdot)}\left(Q \backslash B^{n}(r)\right)$. So let

$$
v \in C^{\infty}\left(Q \backslash B^{n}(r)\right) \cap W^{1, p(\cdot)}\left(Q \backslash B^{n}(r)\right)
$$

be such that

$$
\|u-v\|_{W^{1, p(\cdot)}\left(Q \backslash B^{n}(r)\right)}<\epsilon / r .
$$

Let $\phi$ have support in $B^{n}(2 r)$, equal 1 in $B^{n}(r)$ and be $(1 / r)$-Lipschitz. We show that $\phi u_{B^{n}(2 r)}+(1-\phi) v$ is a sequence of approximating smooth functions. As before we find that

$$
\begin{aligned}
\| u- & \left(\phi u_{B^{n}(2 r)}+(1-\phi) v\right) \|_{1, p(\cdot)} \\
& \leqslant\left(1+\frac{1}{r}\right)\|u-v\|_{W^{1, p(\cdot)}\left(Q \backslash B^{n}(r)\right)}+\left\|u-u_{B^{n}(2 r)}\right\|_{W^{1, p(\cdot)}\left(B^{n}(2 r)\right)} \\
& \leqslant 2 \epsilon+\left(1+\frac{1}{r}\right)\left\|u-u_{B^{n}(2 r)}\right\|_{L^{p(\cdot)}\left(B^{n}(2 r)\right)}+\|\nabla u\|_{L^{p(\cdot)}\left(B^{n}(2 r)\right)}
\end{aligned}
$$

The last term goes to zero, since $|\nabla u| \in L^{p(\cdot)}\left(B^{n}\right)$. So it remains to estimate the middle term. Using the Poincaré inequality for a fixed exponent and denoting the upper bound of $|u|$ by $u_{0}$ we find that

$$
\begin{aligned}
\left\|u-u_{B^{n}(2 r)}\right\|_{L^{p(\cdot)}\left(B^{n}(2 r)\right)} & \leqslant\left\|u-u_{B^{n}(2 r)}\right\|_{L^{p_{1}\left(B^{n}(2 r)\right)}}+\left\||u|+\left|u_{B^{n}(2 r)}\right|\right\|_{L^{p_{2}(A)}} \\
& \lesssim r\|\nabla u\|_{L^{p_{1}\left(B^{n}(2 r)\right)}}+u_{0}|A|^{1 / p_{2}},
\end{aligned}
$$

where $A$ is the set above the curve $y=\sqrt{x}$. Since

$$
\frac{1}{r}|A|^{1 / p_{2}} \sim r^{3 /\left(2 p_{2}\right)-1} \rightarrow 0
$$

as $r \rightarrow 0$, the claim follows.

Notice that if the cusp in the previous example would have been sharper, than we could have drawn the conclusion for a larger range of exponents. Therefore, the pointier the cusp, the better we know how to handle it!

The example gives rise to two questions:

Question 5.4. When can we approximate a Sobolev function locally with a constant, using a Poincaré inequality or otherwise?

Affirmative answers to the following question would mean that we could exclude problematic points from the interior of the domain, which would greatly widen the applicability of our theorems. Notice that the answer is certainly not always affirmative, even when $E$ is just a single point, as is demonstrated by the example in [24]. This property might be related to results on removability in Sobolev spaces, see e.g., [27]. 
Question 5.5. Suppose that $C(\Omega \backslash E) \cap W^{1, p(\cdot)}(\Omega \backslash E)$ is dense in $W^{1, p(\cdot)}(\Omega \backslash$ $E)$, where $E$ is a small set (in some appropriate sense). Does this imply that $C(\Omega) \cap W^{1, p(\cdot)}(\Omega)$ is dense in $W^{1, p(\cdot)}(\Omega)$ ?

As was mentioned in the introduction, we can also look at variable exponent spaces from a different angle and ask:

Question 5.6. Suppose that $C(\Omega) \cap W^{1, p(\cdot)}(\Omega)$ is dense in $W^{1, p(\cdot)}(\Omega)$. What regularity properties of the Sobolev space does this imply?

Although this question has not hereto received that much attention, some results have been derived under the assumption of density. For instance, Harjulehto, Hästö, Koskenoja and Varonen showed that the density of continuous functions is enough to guarantee that every Sobolev function has a quasicontinuous representative [22, Theorem 5.2]. In [16], Fiorenza states that he uses the assumption that smooth functions are dense in order to prove some weak mean-continuity results. However, he then assumes the more specific conditions of the Edmunds-Rákosník result, and it is unclear to the author whether these latter assumptions are really needed in that paper. In domains in $\mathbb{R}^{n}$ variable exponent Newtonian spaces agree with variable exponent Sobolev spaces when continuous functions are dense, hence results under this assumption can be derived from the metric measure spaces setting, see [23].

\section{Acknowledgemt}

The author would like to thank Petteri Harjulehto and the referee for comments on this manuscript.

\section{References}

[1] Acerbi, E. And Mingione, G.: Regularity results for a class of functionals with non-standard growth. Arch. Ration. Mech. Anal. 156 (2001), $121-140$.

[2] Acerbi, E. And Mingione, G.: Gradient estimates for the $p(x)$ Laplacean system. J. Reine Angew. Math. 584 (2005), 117-148.

[3] Chen, Y., Levine, S. and RaO, M.: Variable exponent, linear growth functionals in image restoration. SIAM J. Appl. Math. 66 (2006), no. 4, 1383-1406.

[4] Cruz-Uribe, D., Fiorenza, A. and Neugebauer, C. J.: The maximal function on variable $L^{p}$ spaces. Ann. Acad. Sci. Fenn. Math. 28 (2003), 223-238. Correction in: Ann. Acad. Sci. Fenn. Math. 29 (2004), 247-249. 
[5] Cruz-Uribe, D. and Fiorenza, A.: Approximate identities in variable $L^{p}$ spaces. Preprint (2004). Available at the web address: http://www.na.iac.cnr.it/rapporti/2004/RT279_04.pdf.

[6] Diening, L.: Riesz potential and Sobolev embeddings on generalized Lebesgue and Sobolev Spaces $L^{p(\cdot)}$ and $W^{k, p(\cdot)}$. Math. Nachr. 268 (2004), $31-43$.

[7] Diening, L.: Maximal function on generalized Lebesgue spaces $L^{p(\cdot)}$. Math. Inequal. Appl. 7 (2004), 245-253.

[8] Diening, L., Hästö, P. And Nekvinda, A.: Open problems in variable exponent Lebesgue and Sobolev spaces. In FSDONA04 Proceedings (Milovy, Czech Republic, 2004), 38-58. Academy of Sciences of the Czech Republic, Prague, 2005.

[9] Diening, L. And RŮŽičKA, M.: Calderón-Zygmund operators on generalized Lebesgue spaces $L^{p(\cdot)}$ and problems related to fluid dynamics. J. Reine Angew. Math. 563 (2003), 197-220.

[10] Edmunds, D. E., Kokilashvili, V. and Meskhi, A.: A trace inequality for generalized potentials in Lebesgue spaces with variable exponents. J. Funct. Spaces Appl. 2 (2004), 55-69.

[11] Edmunds, D. E. And RÁkosník, J.: Density of smooth functions in $W^{k, p(x)}(\Omega)$. Proc. Roy. Soc. London Ser. A 437 (1992), 229-236.

[12] FAN, X.-L.: Some results in variable exponent analysis. 5th ISAAC Proceedings, to appear.

[13] FAn, X.-L. AND Zhang, Q.-H.: Existence of solutions for $p(x)$-Laplacian Dirichlet problem. Nonlinear Anal. 52 (2003), 1843-1852.

[14] FAN, X.-L., ZhaO, Y. AND ZhaO, D.: Compact imbedding theorems with symmetry of Strauss-Lions type for the space $W^{1, p(x)}(\Omega)$. J. Math. Anal. Appl. 255 (2001), 333-348.

[15] Fan, X.-L., Wang, S. And Zhao, D.: Density of $C^{\infty}(\Omega)$ in $W^{1, p(x)}(\Omega)$ with discontinuous exponent $p(x)$. Math. Nachr. 279 (2006), no. 1-2, 142149 .

[16] Fiorenza, A.: A mean continuity type result for certain Sobolev spaces with variable exponent. Commun. Contemp. Math. 4 (2002), 587-605.

[17] Futamura, T., Harjulehto, P., Hästö, P., Mizuta, Y. and ShimoMURA, T.: Variable exponent spaces on metric measure spaces. 5th ISAAC Proceedings, to appear.

[18] Futamura, T., Mizuta, Y. and Shimomura, T.: Sobolev embeddings for Riesz potential space of variable exponent. Math. Nachr. 279 (2006), no. $13-14,1463-1473$.

[19] Harjulehto, P.: Variable exponent Sobolev spaces with zero boundary values. To appear in Math. Bohem. 
[20] Harjulehto, P. ANd Hästö, P.: An overview of variable exponent Lebesgue and Sobolev spaces. In Future trends in geometric function theory, 85-93. Rep. Univ. Jyväskylä Dep. Math. Stat. 92. Univ. Jyväskylä, Jyväskylä, 2003.

[21] Harjulehto, P. And Hästö, P.: A capacity approach to the Poincaré inequality and Sobolev imbeddings in variable exponent Sobolev spaces. Rev. Mat. Complut. 17 (2004), 129-146.

[22] Harjulehto, P., Hästö, P., Koskenoja, M. and Varonen, S.: Sobolev capacity on the space $W^{1, p(\cdot)}\left(\mathbb{R}^{n}\right)$. J. Funct. Spaces Appl. 1 (2003), $17-33$.

[23] Harjulehto, P., Hästö, P. and Pere, M.: Variable exponent Sobolev spaces on metric measure spaces. Funct. Approx. Comment. Math. 36 (2006), 79-94.

[24] Hёstö, P.A.: Counter-examples of regularity in variable exponent Sobolev spaces. In The $p$-harmonic equation and recent advances in analysis, 133-143. Contemp. Math. 370. Amer. Math. Soc., Providence, RI, 2005.

[25] Karlovich, A. Y. And Lerner, A. K.: Commutators of singular integrals on generalized $L^{p}$ spaces with variable exponent. Publ. Mat. 49 (2005), no. $1,111-125$.

[26] Kokilashvili, V. And Samko, S.: Maximal and fractional operators in weighted $L^{p(x)}$ spaces. Rev. Mat. Iberoamericana 20 (2004), 493-515.

[27] Koskela, P.: Removable sets for Sobolev spaces. Ark. Mat. 37 (1999), 291-304.

[28] KovÁČIK, O. And RÁkosník, J.: On spaces $L^{p(x)}$ and $W^{k, p(x)}$. Czechoslovak Math. J. 41(116) (1991), 592-618.

[29] Mingione, G. And Mucci, D.: Integral functionals and the gap problem: sharp bounds for relaxation and energy concentration. SIAM J. Math. Anal. 36 (2005), 1540-1579.

[30] NeKvinda, A.: Hardy-Littlewood maximal operator on $L^{p(x)}\left(\mathbb{R}^{n}\right)$. Math. Inequal. Appl. 7 (2004), 255-266.

[31] Pick, L. AND RŮŽičKA, M.: An example of a space $L^{p(x)}$ on which the Hardy-Littlewood maximal operator is not bounded. Expo. Math. 19 (2001), 369-371.

[32] RŮŽIČKA, M.: Electrorheological fluids: modeling and mathematical theory. Lecture Notes in Mathematics, 1748. Springer-Verlag, Berlin, 2000.

[33] Samko, S.: Denseness of $C_{0}^{\infty}\left(\mathbb{R}^{n}\right)$ in the generalized Sobolev spaces $W^{m, p(x)}\left(\mathbb{R}^{n}\right)$. In Direct and inverse problems of mathematical physics (Newark, DE, 1997), 333-342. Int. Soc. Anal. Appl. Comput. 5. Kluwer Acad. Publ., Dordrecht, 2000. 
[34] Samko, S.: On a progress in the theory of Lebesgue spaces with variable exponent: maximal and singular operators. Integral Transform Spec. Funct. 16 (2005), 461-482.

[35] Sharapudinov, I. I.: On the topology of the space $\mathcal{L}^{p(t)}([0,1])$. Math. Notes 26 (1979), no. 3-4, 796-806. Translation of Mat. Zametki 26 (1979), no. 4, 613-632, 655 .

[36] Zhikov, V. V.: Averaging of functionals of the calculus of variations and elasticity theory. Math. USSR-Izv. 29 (1987), 33-66. Translation of Izv. Akad. Nauk SSSR Ser. Mat. 50 (1986), 675-710, 877.

[37] Zhikov, V.V.: On the density of smooth functions in Sobolev-Orlicz spaces (Russian). In Zap. Nauchn. Sem. S.-Peterburg. Otdel. Mat. Inst. Steklov. (POMI) 310, 2004; Kraev. Zadachi Mat. Fiz. i Smezh. Vopr. Teor. Funkts. 35 [34], 67-81, 226. Translation in J. Math. Sci. (N.Y.) 132 (2006), no. $3,285-294$.

Recibido: 18 de abril de 2005

Revisado: 10 de octubre de 2005

Peter A. Hästö

Department of Mathematical Sciences

University of Oulu

P.O. Box 3000

FI-90014, Finland

peter.hasto@helsinki.fi

http://www.helsinki.fi/analysis/varsobgroup/

The author was supported financially by a Gehring-Finland Postdoctoral Fellowship and by the Finnish Academy of Science and Letters. 